\title{
Features of EBM Online
}

\section{INTRODUCTION}

While it's nice to be able to curl up with a cup of tea and the latest paper issue of EBM, the electronic version has a number of advantages too. Although most subscribers still seem to prefer the paper version, there has been a gradual shift since the journal went online in April 2001. We believe that the electronic version will soon predominate because it has many useful features only possible in electronic form, and we plan to continue the enhancement of the website and its interface. Although it is ever changing, we thought it timely to provide our paper readers with a brief description of the features of the website.

\section{SEARCHING}

The majority of the website-including the search engine-is free. The full text contents can be searched back to January 2000. Searching options include: text word, title word, author, and commentator searches, which can include Booleans (AND, OR, and NOT) and truncations. It helps to read the search tips online, but to illustrate, consider trying to find the article:

McDonald HP, Garg AX, Haynes RB. Interventions to enhance patient adherence to medication prescriptions: scientific review. JAMA 2002;288:2868-79.

Any of the following 3 terms in the "Original Citation Authors" box would find this: McDonald or HAYNES or hayne*. The search is case insensitive (so HAYNES is equivalent to haynes) and the * means any further letters. You could also find it using any of the following terms in the "Word(s) in Title" box: adhere* or adherence. Finally, if you used the "Word(s) in Title or Abstract" box, you would still find this article, but a few dozen others as well. Once you have the article, both the full text article and the printable (pdf) version are available.

As a second example, suppose you are interested in diabetic ulcers generally, and search on the text words: diabet* AND ulcer*. This will bring up 10 citations including:

Mason J, O'Keeffe C, McIntosh A, et al. A systematic review of foot ulcer in patients with type 2 diabetes mellitus. I: Prevention. Diabet Med 1999 Oct;16:801-12.

If you now wanted similar or related articles, then there are several options. Firstly, if you click through to the Full Text, a "contents box" appears on the right hand side. Clicking on "Similar articles found in EBM Online" retrieves 62 further articles. Secondly, the bottom of the box shows that this article occurs in 3 Collections of articles: wound care; drugs: infections; and diabetes. Clicking the last topic shows the 24 articles in the diabetes collection. To see all the Collection topics, go to the Homepage and select Collections, which brings up a list (several screens full) of Collection headings. Some of the collections about drugs have links to the relevant section on the eBNF. Try this page: www.evidence-basedmedicine.com/cgi/collection/drugs: infections

\section{ADDITIONAL FUNCTIONS AND FEATURES}

EBM Online offers more functionality and additional web only material. The additional functions include Email @lerts, an alerting service that emails the new tables of contents direct to your inbox when a new issue goes online (before your paper copy!). You can download citations to your citation manager from the Contents Box of an article. A feature called CiteTrack (currently only available for personal subscribers) is a free service providing email based alerts when new articles matching your search criteria are published on the site, or when designated EBM Online articles are cited by new articles from a designated set of journals.

We also have an Email-a-friend feature, the ability to search across all 360+ HighWire journals, abstracts of articles via CrossRef and Medline, free full text of Editors' choice articles, and most importantly, free access for developing countries to the whole site. You should also register (for free) with the HighWire Portal (http://highwire.stanford.edu), which has a powerful search engine and enhanced search result sorting and display and allows you to search across all the HighWire journals and Medline. It's worth noting that registering with the Portal will allow you to register for CiteTrack alerts from any of the 360+ journals.

The additional web only material includes eLetters, web extras (extra content supplementary to the articles published in the paper journal), talks about evidence-based medicine, and links to other evidence-based resources. To have a look at the talk "Finding Gems in Clinical Research" —a talk on the workings of the EBM journal-go to:

www.evidence-basedmedicine.com/misc/slideshows.shtml. Because of the limitations of the paper version, increasing amounts of additional materials are appearing in the web version only, such as further details of some studies. One important "extra" is fuller descriptions of treatments, particularly for non-pharmaceutical interventions. To have a look at all the current web only extra material on EBM Online, go to: www.evidence-basedmedicine.com/supplemental

We think the current web version considerably enhances the usefulness of the journal, but that this can be further improved. For example, we will be starting a preprint project, where 1 or 2 important papers will be published on the site immediately rather than waiting for the paper version to be published. Even though these papers will initially be non-searchable and non-citable, they will still be available for download.

We would encourage you to take a look and use the additional features (even if just as an index to your paper versions). If you would like further help navigating around EBM Online, or want to send us feedback, please go to www.evidence-basedmedicine.com/cgi/feedback or email Dominic Mitchell (dommitchell@bmjgroup.com).

PAUL GLASZIOU, MBBS, PhD BRIAN HAYNES, MD, PhD Editors

DOMINIC MITCHELL BMJ Journals Web Administrator 